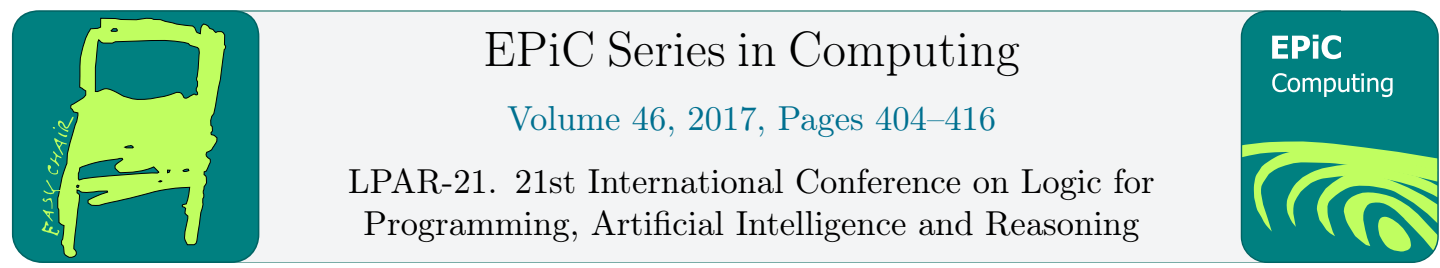

\title{
Gödel logics and the fully boxed fragment of FO-LTL
}

\author{
Matthias Baaz ${ }^{1 *}$ and Norbert Preining ${ }^{2,3}$ \\ 1 Technische Universität Wien, Vienna, Austria \\ baaz@logic at \\ 2 Accelia Inc., Tokyo, Japan \\ 3 Japan Advanced Institute of Science and Technology, Nomi, Japan \\ norbert@preining.info
}

\begin{abstract}
In this paper we show that a very basic fragment of FO-LTL, the monadic fully boxed fragment (all connectives and quantifiers are guarded by $\square$ ) is not recursively enumerable wrt validity and 1-satisfiability if three predicates are present. This result is obtained by reduction of the fully boxed fragment of FO-LTL to the Gödel logic $\mathbf{G}_{\downarrow}$, the infinitely valued Gödel logic with truth values in $[0,1]$ such that all but 0 are isolated. The result on 1-satisfiability is in no way symmetric to the result on validity as in classical logic: this is demonstrated by the analysis of $\mathbf{G}_{\uparrow}$, the related infinitely-valued Gödel logic with truth values in $[0,1]$ such that all but 1 are isolated. Validity of the monadic fragment with at least two predicates is not recursively enumerable, 1-satisfiability of the monadic fragment is decidable.
\end{abstract}

\section{Introduction}

Among the many extensions of classical logic, temporal logics are of particular interest to computer science. Since their introduction in the 1960s by A.N. Prior [15] many different temporal logics have been studied (see, e.g., [9] for an overview); but those referring to a discrete, linear, and well-founded order of time points are of particular relevance to the theory of computation. The main temporal logic of this type is the one in which the set of successive time points is order isomorphic to the natural numbers $\omega$ and where the classical language is extended by (at least) the modal operators $\bigcirc$ and $\square$, which stand for 'at the next time point' and 'from now on always', respectively. This logic LTL is often referred to as the temporal logic of programs (see, e.g., $[1,12]$ ).

In this paper we consider a fragment of the first-order extension of LTL, obtained by fully boxing every component. We show that both validity and satisfiability in this weak fragment is not recursively enumerable. This result is obtained by embedding certain Gödel logics into the fragment.

First order Gödel Logics are a countable family of infinite valued logics where the set of truth values are closed sets $\{0,1\} \subseteq V \subseteq[0,1]$. They are intermediate logics, that is, intermediate

*Research supported by FWF grants P26976-N25 \& I2671-N35 
between classical logic and intuitionistic logic. In fact their propositional fragments are the historically first formulation of intermediate logics introduced by Gödel [10]. In addition, they can be considered as a basis of order based reasoning with uncertainty.

For applications, 1-satisfiability, respectively satisfiability $>0$ (i.e., algorithms for detecting the complements of 1 -satisfiability, respectively $>0$-satisfiability, that is validity of $\neg A$ ) are essential, but contrary to classical logic, validity and non-satisfiability are not dual.

\section{Preliminaries}

We refer the reader to [6] for a detailed introduction to Gödel logics, and recall only the necessary definitions here. Let us first define syntax and semantics of Gödel logics. We adapt some basic notions from [8].

Definition 1 (Gödel set). A Gödel set is any closed set of real numbers, $V \subseteq[0,1]$ which contains 0 and 1 .

Definition 2 (g-embedding). A g-embedding $h$ from a Gödel set $V_{1}$ to a Gödel set $V_{2}$ is an embedding $h: V_{1} \rightarrow V_{2}$ which is continuous (on $V_{1}$ in the usual topology of closed sets on $\mathbb{R}$ ), strictly monotone, and preserves 0 and 1 .

The (propositional) operations on Gödel sets which are used in defining the semantics of Gödel logics have the property that they are projecting, i.e., that the operation uses one of the arguments (or 1) as result (we dot the operators to distinguish them from the syntactical elements of the language introduced later):

Definition 3 (operations on Gödel sets). For $a, b \in[0,1]$ let $a \dot{\wedge} b:=\min (a, b), a \dot{\vee} b:=$ $\max (a, b)$,

$$
a \rightarrow b:= \begin{cases}1 & \text { if } a \leq b \\ b & \text { otherwise }\end{cases}
$$

We define $\dot{\neg} a:=(a \dot{\rightarrow} 0)$, so $\dot{\neg} 0=1$, and $\dot{\neg} a=0$ for all $a>0$. Furthermore, we define $a \dot{\prec} b:=((b \rightarrow a) \rightarrow b)$, which gives 1 iff $a<b$ or $b=1$. This is the closest approximation of strictly less definable in Gödel logics.

For the following let us fix a countable first-order language $\mathscr{L}$ including $\perp$, with the usual definition of $\neg A:=A \rightarrow \perp$. The fact that the language is countable is essential. Note also that we do not include function symbols.

The semantics of Gödel logics with respect to a fixed Gödel set as truth value set and $\mathscr{L}$, is defined using the extended language $\mathscr{L}^{M}$, where $M$ is a universe of objects. $\mathscr{L}^{M}$ is $\mathscr{L}$ extended with symbols for every element of $M$ as constants, so called $M$-symbols. These symbols are denoted with the same letters.

Definition 4 (Semantics of Gödel logic). Fix a Gödel set $V$. A valuation $\varphi$ into $V$ consists of

1. a nonempty set $M^{\varphi}$ (often written as $M$ ), the 'universe' of $\varphi$,

2. for each $k$-ary predicate symbol $P$, a function $P^{\varphi}: M^{k} \rightarrow V$.

Given a valuation $\varphi$, we can naturally define a value $\varphi(A)$ for any closed formula $A$ of $\mathscr{L}^{M}$. For atomic formulas $A=P\left(m_{1}, \ldots, m_{n}\right)$, we define $\varphi(A)=P^{\varphi}\left(m_{1}, \ldots, m_{n}\right)$, and for composite 
formulas $A$ we define $\varphi(A)$ naturally by:

$$
\begin{aligned}
\varphi(\perp) & =0 \\
\varphi(A \wedge B) & =\varphi(A) \dot{\wedge} \varphi(B) \\
\varphi(A \vee B) & =\varphi(A) \dot{\vee} \varphi(B) \\
\varphi(A \rightarrow B) & =\varphi(A) \dot{\rightarrow} \varphi(B) \\
\varphi(\forall x A(x)) & =\inf \{\varphi(A(m)): m \in M\} \\
\varphi(\exists x A(x)) & =\sup \{\varphi(A(m)): m \in M\}
\end{aligned}
$$

(Here we use the fact that our Gödel sets $V$ are closed subsets of $[0,1]$, in order to be able to interpret $\forall$ and $\exists$ as inf and sup in V.)

For any closed formula $A$ and any Gödel set $V$ we let

$$
\|A\|_{V}:=\inf \{\varphi(A): \varphi \text { a valuation into } V\}
$$

Remark. If $V_{1} \subseteq V_{2}$, there are more valuations into $V_{2}$ than into $V_{1}$. Hence $\|A\|_{V_{1}} \geq\|A\|_{V_{2}}$ for all closed $A$.

Similarly, for any map $h: V_{1} \rightarrow V_{2}$, any valuation $\varphi_{1}$ into $V_{1}$ induces a valuation $\varphi_{2}$ into $V_{2}$ as follows:

$$
M^{\varphi_{1}}=M^{\varphi_{2}}, \quad P^{\varphi_{2}}(\vec{m})=h\left(P^{\varphi_{1}}(\vec{m})\right) .
$$

If $h: V_{1} \rightarrow V_{2}$ is a g-embedding from $V_{1}$ into $V_{2}$, and if $\varphi_{2}$ is the valuation induced by $\varphi_{1}$ and $h$, then it is easy to verify by induction on the complexity of the closed formula $A$ that $\varphi_{2}(A)=h\left(\varphi_{1}(A)\right)$, and hence

$$
h\left(\|A\|_{V_{1}}\right) \geq\|A\|_{V_{2}}
$$

for all closed formulas $A$.

Definition 5 (Gödel logics based on $V$ ). Let $V$ be a Gödel set. The first order Gödel logic, $\mathbf{G}_{V}$, is the set of all closed formulas of $\mathscr{L}$ such that $\|A\|_{V}=1$.

The set $\mathbf{G}_{V}$ is also written as $\mathrm{VAL}_{V}$.

From the above remark it is obvious that if $h$ is as above or $V_{1} \subseteq V_{2}$, the Gödel logic $\mathbf{G}_{V_{2}}$ is a subset of $\mathbf{G}_{V_{1}}$.

Finally, we only mention that the downward Löwenheim-Skolem holds, a consequence of the countability of the language (see [7], Proposition 2.13):

Proposition 6 (downward Löwenheim-Skolem). For any valuation $\varphi$ (with $M^{\varphi}$ infinite) there is a submodel $\varphi^{\prime}$ with a countable universe $M^{\varphi^{\prime}}$.

Definition 7 (sub-formula). The only sub-formula of an atomic formula $P$ in $\mathscr{L}^{M}$ is $P$ itself. The sub-formulas of $A \star B$ for $\star \in\{\rightarrow, \wedge, \vee\}$ are the subformulas of $A$ and of $B$, together with $A \star B$ itself. The sub-formulas of $\forall x A(x)$ and $\exists x A(x)$ with respect to a universe $M$ are all subformulas of all $A(m)$ for $m \in M$, together with $\forall x A(x)$ (or, $\exists x A(x)$, respectively) itself.

The set of truth values of valuations of sub-formulas of a set of formulas $\Pi$ under a given valuation $\varphi$ is denoted with

$$
\operatorname{Val}(\varphi, \Pi)=\left\{\varphi(B): \exists A \in \Pi: B \text { sub-formula of } A \text { w.r.t. } M^{v}\right\}
$$

Lemma 8. For a (necessarily at most countable) set of formulas $\Pi$ and a given valuation $\varphi$, there is a valuation $\varphi^{\prime}$ that agrees with $\varphi$ on all atomic formulas (and thus all formulas), and such that the set $\operatorname{Val}\left(\varphi^{\prime}, \Pi\right)$ is at most countable: $\left|\operatorname{Val}\left(\varphi^{\prime}, \Pi\right)\right| \leq \aleph_{0}$. 
Proof. By the downward Löwenheim-Skolem we obtain a countable sub-model where the valuations agree on atomic formulas, and thus also on all formulas. Combined with the countability of the language we obtain the lemma.

In the following we will assume that all valuations under discussion are based on countable models, and thus all Val are always countable, too.

\subsection{The $\operatorname{logics} \mathrm{G}_{\downarrow}$ and $\mathrm{G}_{\uparrow}$}

In the following we will be mainly concerned with the Gödel logics defined by the following two truth value sets:

$$
\begin{aligned}
& V_{\uparrow}=\{1-1 / n: n \geq 1\} \cup\{1\} \\
& V_{\downarrow}=\{1 / n: n \geq 1\} \cup\{0\}
\end{aligned}
$$

From the remark following Definition 4 it follows that every truth value set that has exactly one accumulation point at 1 gives rise to the same Gödel logic, and similar for exactly one accumulation point at 0 . Henceforth, we will indicate with $\mathbf{G}_{\uparrow}$ the logic $\mathbf{G}_{V_{\uparrow}}$, and with $\mathbf{G}_{\downarrow}$ the $\operatorname{logic} \mathbf{G}_{V_{\downarrow}}$.

Previous work on the relation between the logic $\mathbf{G}_{\downarrow}$ and some temporal logics can be found in $[3,4]$. We conclude this section with a statement on the relation between different Gödel logics:

Proposition 9. Intuitionistic predicate logic IL is contained in all first-order Gödel logics.

As a consequence of this proposition, we will be able to use any intuitionistically sound rule and intuitionistically valid formula when working in any of the Gödel logics.

We now also know that $\mathbf{G}_{\uparrow} \neq \mathbf{G}_{\downarrow}$. In fact, we have $\mathbf{G}_{\downarrow} \subsetneq \mathbf{G}_{\uparrow}$; this follows from the following theorem, where $\mathbf{G}_{n}$ indicates the finite valued Gödel logic with $n$ truth values.

Theorem 10 (see [7], Theorem 23).

$$
\mathbf{G}_{\uparrow}=\bigcap_{n \geq 2} \mathbf{G}_{n}
$$

Corollary 11. $\mathbf{G}_{n} \supsetneq \bigcap_{n} \mathbf{G}_{n}=\mathbf{G}_{\uparrow} \supsetneq \mathbf{G}_{\downarrow} \supsetneq \mathbf{G}_{\mathbb{R}}=\bigcap_{V} \mathbf{G}_{V}$

\section{$2.2 \quad$ First Order Linear Time Logic FO-LTL}

Linear Time Logic LTL is the propositional logic over the language $L_{T}$ with the classical connectives $\wedge, \vee, \rightarrow, \leftrightarrow, \neg$, the truth constant $T$, and at least two unary modal operators: the standard temporal connectives $\square$ ('henceforth always') and $\bigcirc$ ('next time'). Note that as we are concerned with a fragment of (FO-)LTL, we do not consider any 'past time' connectives.

Semantically a temporal logic is characterized by a class of partially ordered sets $(T, \leq)$ called time frames. We only consider discrete linear time with a fixed first time point $0 \in T$, and reversed discrete linear time. Standard time frames of these types are those that are order isomorphic to the natural numbers $\omega$ and $\omega^{*}$ (inversely ordered).

FO-LTL is the first-order version of LTL, adding predicates and quantifiers in the same way as when going from classical propositional logic to first order. 
Definition 12 (FO-LTL syntax). Atomic formulas are made up from a countable set of predicates using a countable set of variables. Compound formulas are defined via the usual Boolean connectives, the quantifiers $\forall$ and $\exists$, and the temporal operators: $\bigcirc$ (or $\mathbf{X}$, 'next'), $\square$ (or $\mathbf{G}$, 'global'), U ('until'), F ('eventually', 'future'), R ('release').

Semantics of FO-LTL are given by linear frames of order $\omega$, where the logical part is evaluated locally, and the temporal operations connect the worlds. Although we are restricting our attention to $\bigcirc(\mathbf{X})$ and $\square(\mathbf{G})$, in the following definition of semantics for FO-LTL we restrict ourselves to the operators $\bigcirc(\mathbf{X})$ and $U$, and consider the others as definable:

$$
\mathbf{F} A=\top \mathbf{U} A, \quad \mathbf{G} A=\neg(\mathbf{F}(\neg A)), \quad A \mathbf{R} B=\neg(\neg A \mathbf{U} \neg B)
$$

Similarly, we only provide definitions for negation and disjunction as they are functionally complete in classical propositional logic.

Definition 13 (FO-LTL semantics). A model of FO-LTL is a tuple $\mathcal{M}=(U, \rho)$ such that $U$ is the domain, and $\rho$ gives for each predicate a series of subsets of $U$, defining the predicate at instance $i$. Let $\sigma$ be a mapping from free variables to elements of $D$, and define the forcing relation $\mathcal{M}, \sigma, i \vDash A$ as follows:

- $\mathcal{M}, \sigma, i \vDash P\left(x_{1}, \ldots, x_{n}\right) \quad$ if $\left(\sigma\left(x_{1}\right), \ldots, \sigma\left(x_{n}\right)\right) \in(\rho(P))_{i}$

- $\mathcal{M}, \sigma, i \vDash \neg A \quad$ if $\mathcal{M}, \sigma, i \not \models A$

- $\mathcal{M}, \sigma, i \vDash A \vee B \quad$ if $\mathcal{M}, \sigma, i \vDash A$ or $\mathcal{M}, \sigma, i \vDash B$

- $\mathcal{M}, \sigma, i \vDash \exists x A \quad$ if there exists an $u \in U$ such that $\mathcal{M}, \sigma[x \mapsto u], i \vDash A$

- $\mathcal{M}, \sigma, i \vDash \mathbf{X} A \quad$ if $\mathcal{M}, \sigma, i+1 \vDash A$

- $\mathcal{M}, \sigma, i \vDash A \mathbf{U} B \quad$ if there exists $j>i$ such that $\mathcal{M}, \sigma, j \vDash B$, and for all $i \leq k<j$, we have $\mathcal{M}, \sigma, k \vDash A$

A closed formula $A$ is valid in FO-LTL if for all $\mathcal{M}$ and all $i, \mathcal{M}, \emptyset, i \vDash A$

We note that the definition of $\mathbf{G} A(\square A)$ ensures that if $j \geq i$ and $\mathcal{M}, \sigma, i \Vdash A$, then also $\mathcal{M}, \sigma, j \Vdash A$.

Decidability and undecidability results for FO-LTL are naturally of considerable interest, cf the seminal paper [11]. For complexity discussions and finite model property, see [13]. We sharpen the result on the undecidability of the monadic fragment by embedding $\mathbf{G}_{\downarrow}$ in a weak fragment of FO-LTL.

We consider the following (very weak) fragment of FO-LTL, defined inductively:

Definition 14 (FO-LTL ${ }^{\square}$ ). For every atomic formula $A, \square A \in$ FO-LTL $^{\square}$. If $A$ and $B$ are in FO-LTL ${ }^{\square}$, then $\square(A \star B) \in$ FO-LTL $^{\square}$ where $\star \in\{\wedge, \vee, \rightarrow\}$. If $A(x) \in$ FO-LTL $^{\square}$, then $\square Q x A x$ for $Q \in\{\forall, \exists\}$.

In the following we will identify an evaluation of a formula $A$ in an FO-LTL model $\mathcal{M}$ with an infinite string $\mu(A)$ over the alphabet $\{0,1\}$ :

$$
(\mu(A))_{i}= \begin{cases}1 & \text { if } \mathcal{M}, \emptyset, i \Vdash A \\ 0 & \text { otherwise }\end{cases}
$$

Lemma 15. Any possible valuation of a formula $A$ from FO-LTL ${ }^{\square}$ is either constant $0(\mu(A)=$ $\left.0^{*}\right)$, or finitely many 0 followed by only $1\left(\mu(A)=0^{n} 1^{*}\right.$ with $\left.n \geq 0\right)$.

Proof. The proof is by induction on the recursive definition of the fragment. For atomic $A$, we have that if $i \leq j$ and $\mathcal{M}, \sigma, i \Vdash A$, then also $\mathcal{M}, \sigma, j \Vdash A$. Thus, valuations of $\square A$ are obviously of this form. Any logical connective is guarded by a $\square$, so the statement holds for all formulas from the fragment. 
It is worth noting that although we have added guarding boxes in front of all sub-formulas, it is in fact only necessary for our results to do in front of atomic formulas and implication, as a simple observation shows.

\section{$2.3 \quad$ FO-LTL ${ }^{\square}$ and $\mathrm{G}_{\downarrow}$}

Definition 16 (Translation). The inductive definition of FO-LTL ${ }^{\square}$ gives rise to a bidirectional translation between formulas in the fragment FO-LTL ${ }^{\square}$ and formulas in the language of Gödel Logics:

$$
.^{g}: \text { FO-LTL }^{\square} \mapsto \mathscr{L}_{\mathbf{G}_{\downarrow}} \quad .{ }^{\square}: \mathscr{L}_{\mathbf{G}_{\downarrow}} \mapsto \text { FO-LTL }^{\square}
$$

$A^{g}$ is obtained from $A \in$ FO-LTL ${ }^{\square}$ by dropping all occurrences of $\square$, while $A^{\square}$ is obtained from $A \in \mathscr{L}_{\mathbf{G}_{\downarrow}}$ by putting $\square$ in front of every quantifier and logical connective occurrence.

Example. The formulas

$$
\square \forall x \square \forall y(\square(\square P x \vee \square Q y) \rightarrow \square \exists z \square P z)
$$

and

$$
\forall x \forall y((P x \vee Q y) \rightarrow \exists P z)
$$

are translations of each other.

Lemma 17. There is a mapping $\lambda$ from models of FO-LTL to models of $\mathbf{G}_{\downarrow}$, such that for all $A \in \mathrm{FO}_{\mathrm{LTL}}^{\square}$ and all $B \in \mathscr{L}_{\mathbf{G}_{\downarrow}}$ the computation of truth value commutes with the translation of formulas given by.$^{g}$ and.$^{\square}$, i.e.,

$$
\begin{aligned}
\lambda\left(\varphi_{\mathrm{FO}-\mathrm{LTL}}\right)(A) & =\varphi_{\mathbf{G}_{\downarrow}}\left(A^{g}\right) \\
\lambda^{-1}\left(\varphi_{\mathbf{G}_{\downarrow}}(B)\right) & =\varphi_{\mathrm{FO}-\mathrm{LTL}}\left(B^{\square}\right)
\end{aligned}
$$

Any model of FO-LTL can be seen as a model for $\mathbf{G}_{\downarrow}$ such that the valuations for formulas $A$ from FO-LTL ${ }^{\square}$ and their translations $A^{g}$ evaluate to the same values.

Proof. We have seen in Lemma 15 that the possible valuations $\mu(A)$ for formulas in FO-LTL ${ }^{\square}$ are either constant 0 , that is $\mu(A)=0^{*}$, or a finite sequence of 0 followed by only 1 , i.e., $\mu(A)=0^{n} 1^{*}$. We collect these possible valuations (words over $\{0,1\}$ ) in a set $V$, and order them reversely by the length of the strings of 0 . That is, the biggest element is the one constant 1 , followed by the one with one starting 0 etc:

$$
1^{*}>01^{*}>\cdots>0^{n} 1^{*}>\cdots>0^{*}
$$

This set is order-theoretic isomorph to $V_{\downarrow}$, and we indicate the mapping with $\lambda$. By induction on formulas one can easily show that that for any formula $A \in \mathrm{FO}^{-\mathrm{LTL}^{\square}}$ and each formula $B \in$ $\mathscr{L}_{\mathbf{G}_{\downarrow}}$, we have

$$
\begin{aligned}
\lambda\left(\varphi_{\mathrm{FO}-\mathrm{LTL}}(A)\right) & =\varphi_{\mathbf{G}_{\downarrow}}\left(A^{g}\right) \\
\lambda^{-1}\left(\varphi_{\mathbf{G}_{\downarrow}}(B)\right) & =\varphi_{\mathrm{FO}-\mathrm{LTL}}\left(B^{\square}\right)
\end{aligned}
$$

and thus we can associate with any model of FO-LTL a model of $\mathbf{G}_{\downarrow}$. Furthermore, every model of $\mathbf{G}_{\downarrow}$ is represented by (several) models of FO-LTL.

As a consequence of the previous lemma, we can consider the Gödel logic $\mathbf{G}_{\downarrow}$ as part of FO-LTL. 


\section{Results on validity}

Theorem 18. Validity in $\mathbf{G}_{\downarrow}$ and in $\mathbf{G}_{\uparrow}, \mathrm{VAL}_{\mathbf{G}_{\downarrow}}$ and $\mathrm{VAL}_{\mathbf{G}_{\uparrow}}$, are not decidable.

Proof. In [14] it was shown that the theory of two equivalence relations is recursively inseparable from the theory of two equivalence relations over finite sets, and thus both the theory of two equivalence relations C2E (already shown by Rogers [16]), as well as the theory of two equivalence relations on finite sets $\mathbf{C 2 E F}$ are undecidable.

We first give a faithful interpretation $\tau$ of $\mathbf{C 2 E F}$ in the monadic fragment of $\mathbf{G}_{\downarrow}$ and $\mathbf{G}_{\uparrow}$ by translating counter examples. This shows that $A \in \mathbf{C 2 E F}$ iff $\tau(A) \in \mathbf{G}_{\downarrow}$, and the same for $\mathbf{G}_{\uparrow}$. The translation is the same for both logics.

We now give the translation $\tau$, and assume in the following that the two equivalence relations of $\mathbf{C 2 E F}$ are given by $\equiv_{i}$ for $i=1,2$.

Let us start with $A$ in the language of $\mathbf{C 2 E F}$, and without loss of generality assume that $A$ is already in prenex form with negations removed by forming implications, i.e.,

$$
A=Q_{1} \ldots Q_{n} \bigwedge_{k}\left(\left(\bigwedge_{i} u_{k}^{i} \equiv_{p_{i}} v_{k}^{i}\right) \rightarrow\left(\bigvee_{j} u_{k}^{j} \equiv_{q_{j}} v_{k}^{j}\right)\right)
$$

where the $Q_{i}$ are quantifier terms $\forall u$ or $\exists u$ for the variables $u$ occurring in the formula, and $p_{i}, q_{j} \in\{1,2\}$. With $P_{1}$ and $P_{2}$ being monadic predicate symbols, let

$$
\tau_{1}(A)=Q_{1} \ldots Q_{n} \bigwedge_{k}\left(\left(\bigwedge_{i} P_{p_{i}}\left(u_{k}^{i}\right) \leftrightarrow P_{p_{i}}\left(v_{k}^{i}\right)\right) \rightarrow M \vee\left(\bigvee_{j} P_{q_{j}}\left(u_{k}^{j}\right) \leftrightarrow P_{q_{j}}\left(v_{k}^{j}\right)\right)\right)
$$

where

$$
M=\bigvee_{u}\left(P_{1}(u) \vee P_{2}(u)\right)
$$

where $u$ ranges over all variables $u_{k}^{i}$ and $v_{k}^{i}$, that is, those appearing in $\left(\bigwedge_{i} u_{k}^{i} \equiv_{p_{i}} v_{k}^{i}\right)$.

Let $\tau_{2}(A)$ be the prenex form of

$$
\tau_{1}(A) \vee \exists x P_{1}(x) \vee \exists x P_{2}(x)
$$

Finally, define

$$
\tau(A)=\left(\forall x \neg \neg P_{1}(x) \wedge \forall x \neg \neg P_{2}(x)\right) \rightarrow \tau_{2}(A) .
$$

First assume that $A$ is not valid in $\mathbf{C 2 E F}$, that is, there is a model $\mathcal{M}_{\mathbf{C 2 E F}}$ that evaluates $A$ to false. Assume that within $\mathcal{M}_{\mathbf{C 2 E F}}$ there are $N_{i}$ equivalence classes with respect to $\equiv_{i}$, $i=1,2$. Furthermore, we will denote with $U$ the universe of object of $\mathcal{M}$.

Being a counter-example of $A$ in $\mathbf{C 2 E F}$ means that for each $k$, all the equivalences on the left of the implication must be true, and all the ones on the right side must be false. That is, for all $k$, all $i$ and all $j, u_{k}^{i}$ and $v_{k}^{i}$ must be in the same equivalence class, and all the $u_{k}^{j}$ and $v_{k}^{j}$ must be in different equivalence classes.

We provide a model $\mathcal{M}_{\mathbf{G}_{\downarrow}}$ of $\mathbf{G}_{\downarrow}$ and a model $\mathcal{M}_{\mathbf{G}_{\uparrow}}$ of $\mathbf{G}_{\uparrow}$ in the following way: Let $U$ be also the universe of objects for $\mathcal{M}_{\mathbf{G}_{\downarrow}}$ and $\mathcal{M}_{\mathbf{G}_{\uparrow}}$. For all monadic predicate symbols $R$ other than $P_{1}$ and $P_{2}$ we set $\varphi(R(c))=1$ (or any arbitrary value). We choose $N_{1}+N_{2}$ distinct truth values $V_{\equiv}$ in $(0,1) \cap V_{\downarrow}$ and $(0,1) \cap V_{\uparrow}$, respectively, and specify a bijective mapping

$$
\lambda:\left\{[u]_{\equiv_{1}},[u]_{\equiv_{2}}: u \in U_{\mathbf{C 2 E F}}\right\} \mapsto V_{\equiv} .
$$

Such a mapping exists due to the equicardinality of the two sets. 
We set the valuations of $P_{i}$ to the $\lambda$-image:

$$
\varphi\left(P_{i}(u)\right)=\lambda\left([u]_{\equiv_{i}}\right)
$$

From the above we see that for all $k, i, j$,

$$
\varphi\left(P_{p_{i}}\left(u_{k}^{i}\right)\right)=\varphi\left(P_{p_{i}}\left(v_{k}^{i}\right)\right) \quad \varphi\left(P_{p_{j}}\left(u_{k}^{j}\right)\right) \neq \varphi\left(P_{p_{j}}\left(v_{k}^{j}\right)\right)
$$

Let us now check that this model also assigns a truth value $<1$ to $\tau(A)$ :

$\forall x \neg \neg P_{1}(x) \wedge \forall x \neg \neg P_{2}(x)$ - By the definition of valuation of negation, the fact that $\lambda$ maps into $(0,1)$, and Equation 1 for the valuations of $P$, we obtain that this formula evaluates to 1 .

$\exists x P_{1}(x) \vee \exists x P_{2}(x) \quad$ - The supremum of these valuations is a value $<1$, in fact it is $\max V_{\equiv}$.

$P_{p_{i}}\left(u_{k}^{i}\right) \leftrightarrow P_{p_{i}}\left(v_{k}^{i}\right), P_{q_{j}}\left(u_{k}^{j}\right) \leftrightarrow P_{q_{j}}\left(v_{k}^{j}\right)$ - Due to the definition of $\varphi$ on atomic formulas in Equation 1 we see that for the $i$-case (antecedent) we obtain 1 for all factors, and for the $j$-case (succedent) we obtain one of the valuations of $P\left(u_{k}^{j}\right)$ or $P\left(v_{k}^{j}\right)$, each of which is $<1$.

As a consequence we see that $\tau(A)$ evaluates to a value $<1$, and thus $\tau(A)$ is not valid in $\mathbf{G}_{\downarrow}$ nor $\mathbf{G}_{\uparrow}$.

Consider now the reverse direction, that is, we assume that $\tau(A)$ does not hold in a model of $\mathbf{G}_{\downarrow}$ or $\mathbf{G}_{\uparrow}$, i.e., there is a valuation $\varphi_{g}$ such that $\varphi_{g}(\tau(A))<1$, that is

$$
\varphi_{g}\left[\left(\forall x \neg \neg P_{1}(x) \wedge \forall x \neg \neg P_{2}(x)\right) \rightarrow \tau_{2}(A)\right]<1
$$

The antecedent of this formula can only take 0 or 1 (due to the double negation), and thus we have $\varphi_{g}\left(\forall x \neg \neg P_{1}(x) \wedge \forall x \neg \neg P_{2}(x)\right)=1$, i.e., all the valuations of $P_{i}(c)$ are strictly positive. Furthermore, we have $\varphi_{g}\left(\tau_{2}(A)\right)<1$, thus also

$$
\varphi_{g}\left[\tau_{1}(A) \vee \exists x P_{1}(x) \vee \exists x P_{2}(x)\right]<1,
$$

thus each of the three formulas inside evaluate $<1$. For $P_{i}(u)$ we thus obtain that there is a $\rho$ such that $0<\varphi_{g}\left(P_{i}(u)\right) \leq \rho<1$.

We also obtain from the previous equation that $\varphi_{g}\left(\tau_{1}(A)\right)<1$. Inspecting the definition of $\tau_{1}$, consider first one term in the antecedent: $P_{p_{i}}\left(u_{k}^{i}\right) \leftrightarrow P_{p_{i}}\left(v_{k}^{i}\right)$. This evaluates to either 1 or one of the $P$-components (by definition of the valuation of implication). Thus, the whole antecedent evaluates to either 1 or one of the $P_{i}(u)$ where $u$ ranges over all $u_{k}^{i}$ and $v_{k}^{i}$. In the succedent we have the disjunct $\bigvee_{u}\left(P_{1}(u) \vee P_{2}(u)\right)$, which evaluates to the maximum of the $P_{i}(u)$. If we assume that $\tau_{1}(A)$ evaluates to $<1$, necessarily all the components of the antecedent need to evaluate to $=1$, while all the components of the succedent need to evaluate to $<1$.

We define a model $\mathcal{M}_{\mathbf{C 2 E F}}$ of $\mathbf{C 2 E F}$ (in case of $\mathbf{G}_{\uparrow}$ ) and $\mathbf{C 2 E}$ (in case of $\mathbf{G}_{\downarrow}$ ) as follows: The universes for all models coincide, and

$$
\varphi_{c}\left(u \equiv_{i} v\right)= \begin{cases}1 & \text { if } \varphi_{g}\left(P_{i}(u)\right)=\varphi_{g}\left(P_{i}(v)\right) \\ 0 & \text { otherwise }\end{cases}
$$

for $(c, g) \in\left\{\left(\mathbf{C 2 E F}, \mathbf{G}_{\uparrow}\right),\left(\mathbf{C 2 E}, \mathbf{G}_{\downarrow}\right)\right\}$. By the above, every term in the antecedent evaluates to 1 under $\varphi_{\mathbf{C 2 E}}$, and every term in the succedent to 0 and we obtain that $\mathcal{M}_{\mathbf{C 2 E}}$ is a counter model, and $A$ is not valid in $\mathbf{C 2 E}$ and $\mathbf{C 2 E F}$, respectively.

The only need for differentiating between $\mathbf{C 2 E}$ and $\mathbf{C 2 E F}$ is in this counter example construction, as in the case of $\mathbf{G}_{\uparrow}$ we do only have finitely many truth values available. 
Corollary 19. Validity in $\mathbf{G}_{\downarrow}$ and in $\mathbf{G}_{\uparrow}, \mathrm{VAL}_{\mathbf{G}_{\downarrow}}$ and $\mathrm{VAL}_{\mathbf{G}_{\uparrow}}$, are not recursively enumerable.

Proof. Inspection of the proof of Theorem 18 shows that the counter examples in both cases are enumerable, as the number of equivalence classes $N_{1}+N_{2}$ is finite, and thus also the possible positions of valuations for $P_{i}$. Due to the finiteness of the possible valuations we can filtrate the objects of the universe and obtain a finite models (finitely many objects), which are recursively enumerable.

If the counter examples are recursively enumerable, but validity not decidable, we obtain that validity is not recursively enumerable.

Corollary 20. The fully boxed fragment of FO-LTL with only two monadic predicate symbols is not recursively enumerable.

Proof. The embedding of $\mathbf{C 2 E F}$ only required two monadic predicate symbols.

Remark. It can be shown that the remaining case of only one predicate symbol is decidable.

\section{Results on satisfiability}

Although validity and satisfiability is dual in classical logic, this duality does not hold anymore in many-valued logics, thus also not in the $\operatorname{logics} \mathbf{G}_{\downarrow}$ and $\mathbf{G}_{\uparrow}$, or any other Gödel logic.

As generalization of classical satisfiability we introduce the following two different concepts: 1-satisfiability indicating the set of formulas for which there is a valuation assigning 1 to the formula. And non-0 satisfiability indicating the set of formulas for which there is a valuation assigning a non-0 truth value to the formula.

Definition 21 (satisfiability). For a truth value set $V$, the set of formulas that is 1-satisfiable is indicated with

$$
1-\mathrm{SAT}_{V}=\left\{A: \exists \varphi_{V}: \varphi_{V}(A)=1, A \in \mathscr{L}\right\}
$$

The set of formulas that is non- 0 satisfiable is indicated with

$$
0^{*}-\mathrm{SAT}_{V}=\left\{A: \exists \varphi_{V}: \varphi_{V}(A)>0, A \in \mathscr{L}\right\}
$$

Both definitions are natural generalizations of classical satisfiability and are generally considered in the literature.

Lemma 22. For any truth value set $V$, 1-satisfiability and $>0$-satisfiability of the monadic Gödel logic based on $V, \mathbf{G}_{V}$, coincide, 1-SAT $\mathbf{G}_{V}=0^{*}-\mathrm{SAT}_{\mathbf{G}_{V}}$.

Proof. Any formula that is in 1-SAT $V$ is obviously also in $0^{*}-\mathrm{SAT}_{V}$. Assume a formula $A$ is in $0^{*}-\mathrm{SAT}_{V}$, so there is a valuation $\varphi$ such that $\varphi(A)>0$. Consider the set of valuations under $\varphi$ of all sub-formulas of $A, \operatorname{Val}(\varphi, A)$. By using Lemma 8 , this set is a countable set, thus there is an open interval $(a, b)$ with $b<\varphi(A)$ and non of the valuations falls into $(a, b)$. Define a new valuation $\varphi^{\prime}$ as

$$
\varphi^{\prime}(P)= \begin{cases}1 & \text { if } \varphi(P)>b \\ \varphi(A) & \text { otherwise }\end{cases}
$$

for atomic formulas $P$, and extend $\varphi^{\prime}$ to all formulas. By easy induction on formulas we can show that the above holds for all formulas. The only relevant cases are the quantifiers, but due to the selection of the cut-off point both are preserved. Thus, we obtain that $A$ is also 1-satisfiable. 
Theorem 23. 1-satisfiability in $\mathbf{G}_{\downarrow}, 1-\mathrm{SAT}_{\mathbf{G}_{\downarrow}}$, is not decidable.

Proof. As in the proof of Theorem 18 we will faithfully embed C2EF, but this time we have to deal with a set of complications: To show that the to be defined interpretation is faithful, we will translate satisfying models between the two logics. In particular, when translating from $\mathbf{C 2 E F}$ to $\mathbf{G}_{\downarrow}$ we need to provide some set of finite objects to define the two equivalence relations upon. As we have infinitely many truth values in each neighborhood of 1 , we have to enforce a descent to 0 . In particular, we need to select an open interval in $V$ strictly between 0 and 1 , objects with valuations of the equivalence predicates $P_{i}$ within that interval, and use them to define the equivalence relations in $\mathbf{C 2 E F}$.

A naive method would be to use a formula $\exists y \exists z\left(P_{i} z \prec P_{i} y \wedge \ldots\right)$. This approach does not work as our 'strictly less' relation $\prec$ collapses at 1 (see Section 2) and therefore we cannot be sure that $\varphi_{\mathbf{G}_{V}}\left(P_{i} y\right)$ and $\varphi_{\mathbf{G}_{V}}\left(P_{i} z\right)$ are choosen below 1 .

To overcome this problem we use two other predicates $U(x)$ (for upper) and $L(x)$ (for lower) whose valuations are forced to be a decreasing sequence to 0 . This can be expressed by $\neg \forall x U x \wedge \forall x \neg \neg U x$, as the first conjunct says that the infimum of all evaluations of $U$ is 0 , and the second that every valuation of $U$ is bigger than 0 . Furthermore, we add $L x \prec U x$.

The translation of $A$ is defined via the localized translation $\sigma_{x}$ as follows:

$$
\begin{aligned}
& \sigma_{x}(\forall r B)=\forall r\left(P_{1} r \prec L x \vee U x \prec P_{1} r \vee P_{2} r \prec L x \vee U x \prec P_{2} r \vee \sigma_{x}(B)\right) \\
& \sigma_{x}(\exists r B)=\exists r\left(\left(L x \prec P_{1} r \prec U x\right) \wedge\left(L x \prec P_{2} r \prec U x\right) \wedge \sigma_{x}(B)\right) \\
& \sigma_{x}\left(\bigvee_{j} \bigwedge_{k}\left(r_{j}^{k} \equiv_{i} s_{j}^{k}\right)^{l}\right)=\bigvee_{j} \bigwedge_{k} \sigma_{x}\left(\left(r_{j}^{k} \equiv_{i} s_{j}^{k}\right)^{l}\right) \\
& \sigma_{x}\left(r \equiv_{i} s\right)=\left(P_{i} r \leftrightarrow P_{i} s\right)
\end{aligned}
$$

Note that we didn't define a translation of negative formulas as we start from a formula in C2EF where all negations have been rewritten to implications. The translation $\tau(A)$ of the $\mathbf{C 2 E F}$ formula $A$ is defined as:

$$
\begin{aligned}
\tau(A)=\neg \forall x U x \wedge \forall x \neg \neg & U x \wedge \neg \forall x L x \wedge \forall x \neg \neg L x \wedge \forall x(L x \prec U x) \wedge \\
\forall x(U x \vee \forall y[ & \\
& (L y \prec L x \vee U x \prec L y) \wedge(U y \prec L x \vee U x \prec U y) \wedge \\
& \exists w\left(L x \prec P_{1} w \prec U x \wedge L x \prec P_{2} w \prec U x\right) \wedge \\
& \left.\left.\sigma_{x}(A)\right]\right)
\end{aligned}
$$

We first assume that $\varphi_{\mathbf{C 2 E F}}(A)=1$ and we construct an interpretation $\varphi_{\mathbf{G}}$ of $\mathbf{G}_{V}$ in which $\varphi_{\mathbf{G}}(\tau(A))=1$. Assume in the following that the cardinality of the model $U_{\mathbf{C 2 E F}}$ is $N$.

The universe of the model of $\mathbf{G}_{\downarrow}$ is made up from countable many copies of the (finite) universe $U_{\mathbf{C 2 E F}}$, and an additional countable set of objects $c_{n}$ that are used for the decreasing sequence $U c_{n}$ to 0 :

$$
U_{\mathbf{G}}=\left\{u^{n}: u \in U_{\mathbf{C 2 E F}}, n \in \mathbb{N}\right\} \cup\left\{c_{n}: n \in \mathbb{N}\right\},
$$

where all the $u^{n}$ and $c_{n}$ are different. Note that we add the index $n$ as superscript to $u$ to indicate copies of the elements $u \in U_{\mathbf{C 2 E F}}$.

The values of $U$ and $L$ under $\varphi_{\mathbf{G}}$ meet the following requirements:

(i) $\varphi_{\mathbf{G}}\left(U c_{n+1}\right)<\varphi_{\mathbf{G}}\left(L c_{n}\right)<\varphi_{\mathbf{G}}\left(U c_{n}\right)$,

(ii) $\lim _{n \rightarrow \infty} \varphi_{\mathbf{G}}\left(U c_{n}\right)=0$,

(iii) $\left(\varphi_{\mathbf{G}}\left(L c_{n}\right), \varphi_{\mathbf{G}}\left(U c_{n}\right)\right) \cap V$ has cardinality $N$, and 
(iv) $\varphi_{\mathbf{G}}\left(U u^{n}\right)=\varphi_{\mathbf{G}}\left(L u^{n}\right)=1$ for all $n$ and $u \in U_{\mathbf{C 2} \mathbf{E F}}$.

This definition makes sure that $\varphi_{\mathbf{G}}((3 \mathrm{a}))$ and $\varphi_{\mathbf{G}}((3 \mathrm{c}))=1$.

From the fact that 0 is an accumulation point in $V$, it follows that below any given $\varphi_{\mathbf{G}}\left(U c_{n}\right)$ there exists an open interval containing exactly $N$ truth values. Define $f: \mathbb{N} \rightarrow \mathbb{N}$ and $K_{n}$ such that the following conditions hold: (a) $f$ is strictly monotone increasing, and (b) the open interval $K_{n}=\left(\varphi_{\mathbf{G}}\left(L c_{f(n)}\right), \varphi_{\mathbf{G}}\left(U c_{f(n)}\right)\right)$ contains exactly $N$ truth values. As a consequence of (a) and (b) we have (c) the intervals $K_{n}$ are all disjoint.

Since there are at exactly $N$ truth values in each $K_{n}$ there is for any $n$ an injection $\lambda_{n}$ : $\left\{[u]_{1},[u]_{2}: u \in U_{\mathbf{C 2 E F}}\right\} \rightarrow K_{n} \cap V$. The valuation of $P_{i}\left(u^{n}\right)$ is then defined as

$$
\varphi_{\mathbf{G}}\left(P_{i}\left(u^{n}\right)\right)=\lambda_{n}\left([u]_{i}\right) .
$$

This ensures that (3d) is satisfied. To complete the definition of the valuation of atomic formulas we set $\varphi_{\mathbf{G}}\left(P_{i}\left(c_{n}\right)\right)=1$ for all $n$.

We will now show that $\varphi_{\mathbf{G}}(\tau(A))=1$. Consider the universal quantifier $\forall x$ in (3b) and pick up an arbitrary element $x$ from $U_{\mathbf{G}}$. If $x=u^{n}$ then $\varphi_{\mathbf{G}}(P x)=1$ (see (iv) above). Otherwise, that is when $x=c_{n}$, what remains to show is that $\varphi_{\mathbf{G}}\left(\sigma_{x}(A)\right)=1$. We can indeed give a selection function for the existentially quantified variables within $\sigma_{x}(A)$ : If all the quantifiers in front of an existential quantifier are instantiated, simply drop all the super-scripts in $u^{n}$, consider the resulting assignment in $\mathbf{C 2 E F}$, and use the object selected by the existential quantifier there, adding the index of the current interval in which we evaluate. Therefore the existential quantifiers are always evaluated in the current interval, and thus the first two conjuncts of (2b) are satisfied.

On the other hand considering the universal quantifier and (2a) we see that if the object instantiating the universal quantifier is outside the current interval, i.e., the valuations are outside the interval defined by $\varphi_{\mathbf{G}}(L x)$ and $\varphi_{\mathbf{G}}(U x)$, the evaluation of $(2 \mathrm{a})$ immediately becomes 1 .

So we can assume in the following that all objects instantiating quantified variables in (3e), i.e., in $\sigma_{x}(A)$, give valuations of $P_{1}$ and $P_{2}$ within the interval under discussion. So we have $\varphi_{\mathbf{G}}\left(\sigma_{c_{n}}(A)\right)=1$ for all $c_{n}$, and by the definition of $\varphi_{\mathbf{G}}, \varphi_{\mathbf{G}}(\tau(A))=1$.

For the reverse direction assume that $\varphi_{\mathbf{G}}(\tau(A))=1$. For $\varphi_{\mathbf{G}}$ there exists an $c$ such that $0<\varphi_{\mathbf{G}}(P c)<1$, as $\varphi_{\mathbf{G}}((3 \mathrm{a}))=1$. Define the universe of $\varphi_{\mathbf{C 2 E}} \mathbf{E}$ as

$$
U_{\mathbf{C 2 E F}}=\left\{u \in U_{\mathbf{G}}: \varphi_{\mathbf{G}}(L c)<\varphi_{\mathbf{G}}\left(P_{i} u\right)<\varphi_{\mathbf{G}}(U c), i=1,2\right\}
$$

Note that $U_{\mathbf{C 2 E F}}$ cannot be empty as $\varphi_{\mathbf{G}}((3 \mathrm{~d}))=1$, and since $\varphi_{\mathbf{G}}(L(c))>0$, that it also contains only finitely many values. Define a valuation $\varphi_{\mathbf{C 2 E F}}$ as

$$
\varphi_{\mathbf{C 2 E F}}\left(a \equiv_{i} b\right)=1 \quad \text { iff } \quad \varphi_{\mathbf{G}}\left(P_{i} a \leftrightarrow P_{i} b\right)=1
$$

from which follows that $\varphi_{\mathbf{C 2 E F}}(A)=1$ being $\varphi_{\mathbf{C 2} \mathbf{E F}}(A)$ nothing but the valuation $\varphi_{\mathbf{G}}\left(\sigma_{c}(A)\right)$.

Corollary 24. With the notions from the previous proof, the set

$$
\{\tau(A): \exists \varphi \varphi(\tau(A))=0\}
$$

is recursively enumerable.

Proof. If $\varphi_{\mathbf{C 2 E F}}$ is a counterexample for a formula $A$ in $\mathscr{L}_{\mathbf{C 2 E F}}$, then the constructed valuation $\varphi_{\mathbf{G}_{\downarrow}}$ will evaluate the translated formula $\tau(A)$ to 0 . The counter-examples in C2EF are recursively enumerable, thus also the above set. 
Theorem 25. The set of not 1-satisfiable formulas of $\mathbf{G}_{\downarrow},\left(1-\mathrm{SAT}_{\mathbf{G}_{\downarrow}}\right)^{c}$, is not recursively enumerable.

Proof. Assume that (1-SAT $\left.\mathbf{G}_{\downarrow}\right)^{c}$ is recursively enumerable. From Lemma 22 we know that $1-\mathrm{SAT}_{\mathbf{G}_{\downarrow}}$ and $0^{*}-\mathrm{SAT}_{\mathbf{G}_{\downarrow}}$ coincide, so $\left(0^{*}-\mathrm{SAT}_{\mathbf{G}_{\downarrow}}\right)^{c}$ is recursively enumerable. This implies that

$$
\{A: \varphi(A)=0 \text { for all valuations }\}
$$

is also recursively enumerable.

We are switching now back to FO-LTL ${ }^{\square}$ and obtain that

$$
\left\{\text { FO-LTL } \Vdash \neg B: B \in \text { FO-LTL }^{\square}\right\}
$$

is recursively enumerable. Note that while $B \in \mathrm{FO}^{-\mathrm{LTL}^{\square}}, \neg B \notin \mathrm{FO}^{\square} \mathrm{LTL}^{\square}$.

Due to the fact that $B \in \mathrm{FO}^{-\mathrm{LTL}^{\square}}$, we see that

$$
\left\{\text { FO-LTL } \Vdash \neg \square(B \rightarrow \square \perp): B \in \text { FO-LTL }^{\square}\right\}
$$

is also recursively enumerable, as it is a sub-case of the previous class.

Using the valid equivalence of $\square \perp \leftrightarrow \perp$ we obtain that

$$
\left\{\text { FO-LTL } \Vdash \neg \square \neg B: B \in \text { FO-LTL }{ }^{\square}\right\}
$$

is recursively enumerable. This means, that the valuations of $B$ are not constant 0 . Reading that back in Gödel logics we obtain that

$$
\{A: \varphi(A)>0 \text { for all valuations }\}
$$

is also recursively enumerable. Combining this fact with Corollary 24 that

$$
S=\{\tau(A): \exists \varphi \varphi(\tau(A))=0\}
$$

is recursively enumerable, we obtain that the set $S$ is also decidable, and thus also C2EF, a contradiction. Thus, the initial assumption that $\left(1-\mathrm{SAT}_{\mathbf{G}_{\downarrow}}\right)^{c}$ is recursively enumerable is false.

\section{Conclusion}

Validity of the monadic fragent in $\mathbf{G}_{\downarrow}$ and $\mathbf{G}_{\uparrow}$ is not recursively enumerable by Corollary 19. In contrast, the quantified propositional logic is decidable (see [5] for $\mathbf{G}_{\downarrow}$ and [2] for $\mathbf{G}_{\uparrow}$ ) Both theories even enjoy quantifier elimination if a 1-placed connective $\star$ is added. ( $\star$ denotes in the predecessor (successor) truth value in $\mathbf{G}_{\downarrow}\left(\mathbf{G}_{\uparrow}\right)$, and is constant on 0 (1)). This quantifier elimination transfers to the corresponding fully boxed fragment of FO-LTL if the operator $\star$ with the meaning $\star A$ is true iff $A$ is true at the next moment is allowed to be added anywhere and several times to the formulas of the fully boxed fragment.

\section{References}

[1] H. Andreká, V. Goranko, S. Mikulás, I. Németi, and I. Sain. Effective temporal logic of programs. In A. Szalas L. Bolc, editor, Time and Logic. A Computational Approach, pages 51-129. UCL Press, 1995. 
[2] M. Baaz, A. Ciabattoni, and R. Zach. Quantified propositional Gödel logics. In Proceedings of LPAR'2000, LNAI 1955, pages 240-257, 2000.

[3] M. Baaz, A. Leitsch, and R. Zach. Completeness of a first-order temporal logic with time-gaps. Theoret. Comput. Sci., 160(1-2):241-270, June 1996.

[4] M. Baaz, A. Leitsch, and R. Zach. Incompleteness of a first-order Gödel logic and some temporal logics of programs. In Hans Kleine Büning, editor, Computer Science Logic, Proc. 9th Workshop, CSL'95, LNCS 1092, pages 1-15. Springer, 1996.

[5] M. Baaz and N. Preining. Quantifier Elimination for Quantified Propositional Logics on Kripke Frames of Type $\omega$. Journal of Logic and Computation, 18(4):649-668, 2008.

[6] M. Baaz and N. Preining. Gödel-Dummett logics. In Petr Cintula, Petr Hájek, and Carles Noguera, editors, Handbook of Mathematical Fuzzy Logic, volume 2, chapter VII, pages 585-626. College Publications, 2011.

[7] M. Baaz, N. Preining, and R. Zach. First-order Gödel logics. Annals of Pure and Applied Logic, 147:23-47, 2007.

[8] A. Beckmann, M. Goldstern, and N. Preining. Continuous Fraïssé conjecture. Order, 25(4):281298, 2008.

[9] E. A. Emerson. Temporal and modal logic. In Handbook of Theoretical Computer Science, volume Volume B: Formal Models and Semantics, pages 995-1072. Elsevier and MIT Press, 1990.

[10] K. Gödel. Zum Intuitionistischen Aussagenkalkül. Ergebnisse eines mathematischen Kolloquiums, 4:34-38, 1933.

[11] I. Hodkinson, F. Wolter, and M. Zakharyaschev. Decidable fragments of first-order temporal logics. Annals of Pure and Applied Logic, 106(1):85-134, 2000.

[12] F. Kröger. Temporal Logic of Programs, volume EATCS Monographs in Computer Science 8. Springer, Berlin, 1987.

[13] D. Kuperberg, J. Brunel, and D. Chemouil. On Finite Domains in First-Order Linear Temporal Logic, pages 211-226. Springer International Publishing, Cham, 2016.

[14] I. A. Lavrov. Effective inseparability of the sets of identically true formulae and finitely refutable formulae for certain theories. Algebra i Logika Sem., 2:5-18, 1963. In Russian. MR0157904.

[15] A. N. Prior. Past, present, and future. Oxford University Press, 1967.

[16] H. Rogers. Certain logical reduction and decision problems. Annals of Mathematics, 64:264-284, 1956. 